\title{
Cosmovisión del Pueblo Indígena Mayangna Sauni Arungka en la práctica de la comunicación intercultural para la defensa territorial y ambiental
}

\author{
Worldview of the Mayangna Sauni Arungka Indigenous People in the practice \\ of intercultural communication for territorial and environmental defense
}

\author{
Máximo José Garth Medina ${ }^{1}$ \\ Angélica Leonor Ruiz Calderón ${ }^{2}$
}

\section{Resumen}

Esta investigación sistematiza la manera en que el pueblo indígena Mayangna Sauni Arungka se comunica interculturalmente al momento de defender el territorio y el medio ambiente. Es un estudio cualitativo con una perspectiva holística donde participaron líderes del gobierno territorial, hombres y mujeres del territorio Mayangna Sauni Arungka. La información se recogió a partir de la entrevista, la observación y la revisión documental. El análisis consistió en categorizar a través del procesamiento de una matriz que contribuyó a la triangulación de la información. Los principales resultados muestran que los aciertos sobre la cosmovisión del pueblo indígena Mayangna se enfocan en una comunicación intercultural, para la defensa territorial, la conservación y preservación de los recursos naturales; y que la comunicación oral es un instrumento para el intercambio de conocimientos, saberes y prácticas. Por lo tanto, se hace necesario crear y fortalecer capacidades dentro del territorio por medio de la formación para promover los conocimientos, saberes, capacidades, valores, principios en equilibrio y armonía con la Madre Tierra.

Palabras clave: Pueblo indígena mayangna; saberes; buenas prácticas; comunicación intercultural; cosmovisión; diálogo.

\section{Abstract}

This research systematizes the way in which the Mayangna Sauni Arungka indigenous people communicate interculturally among themselves, when defending the territory and the environment. It is a qualitative study with a holistic perspective involving territorial government leaders, men and women of the Mayangna Sauni Arungka territory. The information was collected from the interview, observation and documentary review. The analysis consisted of categorizing through the processing of a

\footnotetext{
1 Máster en Comunicación Intercultural, Divulgador de URACCAN en el Recinto Universitario Las Minas. Correo: jose.garth@uraccan.edu.ni - ORCID: https://orcid.org/oooo-ooo2-5896-9952

2 Doctora en Educación con especialidad en Mediación Pedagógica, Vicerrectora de la Universidad de las Regiones Autónomas de la Costa Caribe Nicaragüense-Recinto Universitario Las Minas. Correo: vicerrectoría.lasminas@uraccan.edu.ni
}

Recibido: 26/02/2018 Aprobado: 28/08/2018 
matrix that contributed to the triangulation of the information. The main results show that the successes on the Mayangna indigenous people's worldview focus on an intercultural communication, for territorial defense, conservation and preservation of natural resources; and that oral communication is an instrument for the exchange of knowledge, knowings and practices. Therefore, it is necessary to create and strengthen capacities within the territory through training to promote knowledge, knowings, skills, values, principles in balance and harmony with Mother Earth.

Keywords: Mayagna indigenous people; knowings; good practices; intercultural communication; worldview; dialogue.

\section{Introducción}

La espiritualidad, conocimientos y pensamientos de los pueblos indígenas de Abya Yala son el origen de sus formas y proyectos de vida. Las características intrínsecas, especialmente los derechos a sus tierras, territorios y recursos, derivan de sus estructuras políticas, económicas, sociales, culturales, tradiciones espirituales, historia y su concepción de la vida. Los pueblos indígenas contribuyen a la riqueza y diversidad de las civilizaciones y culturas.

Diversos instrumentos internacionales y nacionales de protección de los derechos humanos reconocen que, el respeto de los conocimientos, las culturas y las prácticas tradicionales indígenas contribuyen al desarrollo sostenible y equitativo, así como al ordenamiento del medio ambiente. Los estudios sobre esos conocimientos, sin embargo, se han hecho generalmente desde la posición de la ciencia occidental, que ha puesto en situación de subordinación los conocimientos indígenas (Cuningham, 2008).

En las Regiones Autónomas de la Costa Caribe de Nicaragua existen 6 grupos étnicos, entre ellos dos pueblos indígenas: Miskitus y Mayangnas, éste último con tres variantes lingüísticas, Tuahka, Panamahka y Ulwas. Los indígenas Mayangna están asentados en las Regiones Autónomas de la Costa Caribe Norte y Sur, así como en la Cuenca del Alto Wangki y Bocay. La población indígena Mayangna se ha visto afectada en los último 20 años con una invasión de sus territorios lo que ha implicado un cambio en sus estilos de vida, es por ello, que este estudio describe la cosmovisión del pueblo indígena Mayangna Sauni Arungka en la práctica de la comunicación intercultural al momento de emprender la defensa territorial y ambiental, asumiendo que la comunicación intercultural ayuda a armonizar el conflicto de invasión territorial y, por ende, disminuir el daño ambiental y ecológico del territorio indígena Sauni Arungka.

\section{Revisión de la literatura}

Cuando se describe a una comunidad humana puede constatarse que posee características propias relacionadas con las formas de obtener la subsistencia, las instituciones, 
la organización del parentesco, los estilos de vida y los sistemas de representaciones. Desde esta perspectiva, la cultura es entendida como un conjunto de rasgos que le son propios, cuyos límites coinciden con los de un grupo humano y se concretan en una determinada área según (Comas-d Argemir, 1998), citado por Ruiz (2010, p.31).

Siguiendo a Taylor y Bogdan (1996), en Ruiz (2010) se entiende la cultura como: [...] aquel todo complejo que incluye el conocimiento, las creencias, el arte, la moral, el derecho, las costumbres y cualesquiera otros hábitos y capacidades adquiridos por el hombre en cuanto miembro de la sociedad [...]

Para Boas (2008), en Ruiz (2010)

[...] la cultura incluye todas las manifestaciones de los hábitos sociales de una comunidad, las reacciones del individuo en la medida en que se ven afectadas por las costumbres del grupo en que vive, y los productos de las actividades humanas en la medida en que se ven determinadas por dichas costumbres [...]

Geertz (1994), enfatiza que para entender cultura en sus propios términos debemos entender sus complejidades, sutilezas y matices. Insta a la interpretación de las culturas, como tituló uno de sus libros, en donde nos esclarece el concepto de cultural al escribir acerca de culturas particulares. Es buscar un significado, una explicación, pero no a partir de leyes, sino argumentada (Álvarez \& Grunberg, 2004, p. 2).

La discusión de los autores nos lleva a plantear que describir una cultura no es sencillo, no se reduce a pensar que sólo es observar como las personas se comportan en una sociedad y luego tomar nota de su comportamiento. Su complejidad pasa por entender que existen comportamientos diversos.

Durkheim (1997, p. 26) hace hincapié y señala que la cultura es algo que está fuera de nosotros y que ejerce un fuerte poder coercitivo sobre las personas. También reconoce que existen limitaciones directas que resultan ser más obvias y las indirectas que menos obvias. En definitiva, la cultura es lo que nos hace diferentes de otros.

Para los indígenas del norte de México la cosmovisión es entendida como un conjunto articulado de saberes y creencias que les permite a los seres humanos, explicarnos el mundo y su propia existencia; así se forma un modelo interpretativo de la realidad, mismo que prescribe, prohíbe y califica las motivaciones y conducta de los individuos en el desarrollo de la práctica social (Diego Prieto \& Alejandro Vásquez, 2004).

Cosmovisión Amazónica es una comunicación que concierne a la visión que los Pueblos de la Amazonía peruana tienen acerca del mundo. Todas las sociedades humanas buscan explicar el universo que las rodea. En las cosmovisiónes indígenas 
amazónicas plantea la existencia de una diversidad de mundos, cada uno con sus propias topografías, habitantes y leyes, y la de una diversidad de esferas al interior del mundo que vivimos (Calero, 2014).

De acuerdo a Silva (2015), la cosmovisión de los Pueblos Indígenas de Bolivia expresa una alternativa necesaria para combatir las diferentes crisis de la humanidad por tanto es considerada como las formas de pensar, de conocimientos y prácticas originarias.

Para poder entender la noción de la comunicación intercultural es esencial partir de la concepción de interculturalidad. La interculturalidad, en tanto dinámica inherente entre culturas, es la oportunidad para aprender mutuamente, lo que para los seres humanos sólo es posible a través del diálogo como la forma cotidiana de relacionarse entre sí. Como concepto y práctica, la interculturalidad significa "entre culturas", pero no simplemente un contacto entre culturas, sino un intercambio que se establece en términos de equidad e igualdad (Walsh, 2005).

Desde esta perspectiva la comunicación intercultural, es un espacio de respeto, diálogo e intercambio de saberes entre los pueblos pertenecientes a culturas distintas que se han intentado comunicar siempre (Rodrigo, 2011).

En definitiva, la comunicación intercultural es una práctica que fortalece derechos y deberes de los pueblos y, transforma los procesos educativos desarrollados en diferentes partes del mundo. Significa oportunidades para acercarnos a demandas sugeridas y propias de la comunidad y la sociedad en su conjunto, en un esfuerzo por lograr que el que recibe información, pueda aportar desde su condición de sujeto, prevaleciendo el respeto a su identidad cultural, territorial, espiritual y económica (URACCAN, 2009).

En relación al conocimiento tradicional, el mundo occidental lo relaciona con mitos y con prácticas que carecen de fundamentos científicos, negando por consiguiente toda posibilidad de que la vida comunitaria gira en torno a una serie de elementos culturales que intervienen en el desarrollo social de cada grupo (Fedrick, 2003).

El conocimiento indígena se define como singular, tradicional y local que existe en el contexto de mujeres y hombres de una sociedad. Abarca todos los aspectos de la vida, inclusive el manejo y las relaciones con el entorno natural. Es un sistema acumulativo y representa generaciones de experiencias, observación cuidadosa y experimentación constante. Los sistemas de conocimiento indígena son dinámicos: continuamente se agregan a éste, nuevos conocimientos. Estos sistemas producen innovaciones desde dentro y también usan y adaptan conocimientos externos a su situación. El conocimiento indígena se guarda en la memoria y en las actividades de las personas y se expresa en cuentos, canciones, folclor, proverbios, danzas, mitos, 
valores culturales, creencias, rituales, leyes comunitarias, lenguaje local y taxonomías, prácticas agrícolas, herramientas, materiales, especies de plantas y animales. (Cunnigham, 2008).

Para los pueblos indígenas, la espiritualidad es energía y acción. Es la esencia que da vida a la materia (los seres humanos, los animales, las plantas y los minerales) y aquí la relación intrínseca con el cosmos, donde se conjugan las fuerzas energéticas de los seres que habitamos esta tierra (sentimientos, pensamientos, acciones). El propósito fundamental de la espiritualidad es la búsqueda del equilibrio y la armonía con nosotros mismos y con los demás (cosmos), la pérdida de estos dos importantes principios ha provocado grandes catástrofes a escala local, regional y mundial.

La espiritualidad indígena tiene varias manifestaciones y medios para lograr el equilibrio, entre ellas: el fuego sagrado, las ceremonias, lugares sagrados, cerros, montañas, ríos, lagos, cantos, ofrendas. La espiritualidad colectiva y las prácticas espirituales de los pueblos logran la fuerza, el equilibrio y la armonía para el buen vivir/vivir bien/ bien-estar comunitario.

A como plantea Cox Molina (2011, p.93), "la práctica de la espiritualidad tiene por sí misma una función social: Restablecer el equilibrio de los desórdenes ocasionados por la transgresión y ruptura a las normas, curar a las enfermedades provocadas por la mala influencias o hechicerías o bien, hacer volver el alma perdida de las personas y velar por la unidad de los pueblos de tradición".

\section{Materiales y métodos}

Esta investigación se desarrolló bajo el paradigma cualitativo, que apunta a entender las situaciones y comportamientos desde la perspectiva subjetiva, es decir, desde el interior. La característica básica de la investigación cualitativa se inclina a comprender los valores, las creencias, las normas a través de la mirada de quienes están sometidos a un estudio. El estudio se realizó desde una perspectiva holística, no reduciendo a variables sino viendo el hecho como un todo y en interrelación con todos sus aspectos. Un elemento fundamental fue el análisis desde la visión de los sujetos y no ideas preconcebidas del investigador.

Con respecto a los métodos cualitativos, se tomó como estrategia para la obtención de los datos, la observación no participante, entrevista a informantes claves y el análisis de documentos. Se realizaron tres sesiones: la primera para la validación del diseño de la investigación, la segunda para la presentación de los resultados preliminares y la tercera la revisión del informe final. Finalmente, con los aportes en estas sesiones se estructuró el informe definitivo donde se establecen los principales hallazgos de este cultivo que finaliza con la propuesta de un proyecto de comunicación a implementar en el territorio. 


\section{Resultados y discusión}

La comunicación intercultural es entendida como una forma de construir el conocimiento, los saberes y pensamientos para favorecer el dialogo o conflicto, por tanto, se constituye como una forma de interacción mutua cultural y vivida desde la práctica del Buen Vivir. También, es entendida como una aspiración de participación, respeto para escucharse entre sí y con los otros.

M. Juwith Francis (2015) señala que los elementos determinantes de la comunicación intercultural se asocian a saberes y creencias, lo que coincide con el planteamiento de Prieto y Vásquez (2004), donde expresa que esta es una forma de entender la cosmovisión desde los Pueblos Indígenas del norte de México. Sin embargo, al asociarlo con el planteamiento de Silva (2015) nos encontramos que los Pueblos Indígenas de Bolivia la asumen como una forma necesaria y alternativa para combatir diferentes crisis de la humanidad.

Si analizamos a Taylor (2015) nos encontramos que un elemento expresivo visible desde la cultura de la comunidad Mayangna Arungka está referida a las creencias y hábitos que prevalece en un pensamiento humano ancestral, la que se constató con añoranza ya que se mantiene en la mente de los abuelos y abuelas aquellas formas ancestrales de mantener la comunicación con el mundo que les rodea, la naturaleza y la Madre Tierra. La creencias que aún viven en la mente de los abuelos y abuelas del territorio dejan muy claro esa interacción que existió antes, porque hoy sólo quedan los recursos de aquellas formas de comunicación que han abandonado, esto coincide con lo que plantea Boas (2008) que señala que la cultura incluye manifestaciones de hábitos sociales de una comunidad, lo que fue evidente en todas las comunidades donde hay iglesias como sus formas de expresión espiritual de hoy, lo cual determina que esa manifestación se cimenta en las costumbres de los pueblos.

Para Martina Juwith Williams (2015) la comunicación está relacionada a la capacidad de nuestros ancestros a la conexión con los espíritus del universo y el más allá, esta connotación esta dimensionada desde los saberes como un elemento cultural que facilita el diálogo desde las prácticas ancestrales que pone de manifiesto el símbolo como medio para identificar escenarios y contextos vinculados a la madre tierra.

Este planteamiento señala la cosmovisión desde una explicación del universo y desde la connotación de los pueblos indígenas Mayangna Panamahka, está relacionado con lo que plantea Calero y Larios Astrid (2014) donde expresan que los pueblos de la Amazonía entienden el mundo desde un entorno que los rodea. De esta manera, se consigue identificar que hay otros elementos que intervienen en la comunicación como es el contexto y los escenarios que se relacionan con la cultura social. 
La relación directa que mantenían los ancestros de los indígenas Mayangnas en el territorio Arungka con la naturaleza y con los espíritus fue una práctica que la mayoría de los sabios y sabias del territorio han dejado claro, al considerar que hubo una gran capacidad de los ancestros de comunicarse con los espíritus y con la madre naturaleza, a ella se le entendía, se le comprendía y siempre hubo una relación armónica entre la Madre Tierras y las poblaciones originarias del Sauni Arungka.

Martina Juwith Bans (2015) señala que la cosmovisión Mayangna Sauni Arungka se establece como una forma de ver, sentir, escuchar, interpretar y ver las señales del territorio, incluyendo percepciones a través de los sueños, destrezas singular y propia de quienes han construido su realidad a través de la oralidad. En este hallazgo se pueden rescatar algunos valores conferidos a la Madre Tierra como un elemento estrictamente ligado a la vida comunitaria de los Pueblos Indígenas que para Fedrick (2003) determina la relación y el valor que se le da a esto desde la cosmovisión indígena.

Por otro lado, los sabios del territorio consideran que la tradición de espiritualidad que había en los ancestros y los grandes poderes sobrenaturales que poseían los Ditalyan, fueron reducidos por la entrada de la religión, cuando son obligados a la conversión y abandonar sus prácticas tradicionales. La comunicación que había con los espíritus, con la naturaleza tuvo que ser abandonada por el cristianismo que tildó todos estos actos como diabólicos, aunque en los pueblos del sur, se resistieron y lograron mantener sus tradiciones. El pueblo Indígena Mayangna fue transculturizado y la evangelización que llegó la religión Morava al Caribe, desterraron por completo estas costumbres ancestrales de este pueblo.

Este planteamiento evidencia que existe una transculturización hacia los pueblos, lo que ha trastocado el derecho a practicar y revitalizar sus tradiciones y costumbres culturales, lo cual no es coincidente con lo que establece la Declaración de la Organización de Naciones Unidas sobre los Derechos de los Pueblos Indígenas. Lo que indica que el cristianismo ha mantenido a los pueblos indígenas sumergidos en un pensamiento ideológico de culturas no originarias.

Lo expresado por los abuelos y abuelas, explicita que la comunicación intercultural desde la cosmovisión indígena es vista como algo natural, pues se consideran pueblos originarios que conservan sus territorios y sus bosques porque de ellos depende su existencia. Al relacionarlo con lo que señala Jefe Joseph (1879), citado por Marín (2017), nos encontramos que la espiritualidad es la energía esencial y acción de los pueblos indígenas. Por eso es que los ancestros tenían esa gran capacidad de comunicarse con los árboles, los ríos, las piedras, los animales, y las plantas que fueron usadas para la curación de enfermedades, lo que indica que la espiritualidad indígena tiene varias manifestaciones y medios para lograr el equilibrio y la armonía con ellos mismos y los demás cosmos. 
"Antes no teníamos los problemas que hoy tenemos en nuestras comunidades con la invasión de los colonos..." Esta afirmación de una de las abuelas del territorio nos indica que el tema de la defensa territorial de los ancestros estaba centrado en regulaciones de caza, pesca y uso del bosque, donde se podía lograr un control por medio de las creencias ancestrales en el sentido que cada uno de los elementos del bosque, animales, flora y fauna tenían sus dueños que podían reclamar si se daba una sobre explotación.

La comunicación intercultural en la cosmovisión indigna Mayangna estaba relacionada a transmitir por medio de la palabra los peligros que representaba para la vida y la sobrevivencia, la caza, la pesca y la tala de los árboles en escala mayor. El respeto inculcado por los ancestros provocó un control sobre el uso de los diferentes recursos naturales que tenían dentro de su territorio.

A manera de ejemplo, los ancestros decían que si una persona que se internaba en las montañas encontraba una manada de jabalíes no podía cazarlos a todos, pues los dueños de los jabalíes se enfadarían y podían causarle mucho daños al cazador, esta teoría que usaron los ancestros era para evitar que se diera una caza a gran escala de esta especie y de esta manera se garantizaba la existencia de este animal como alimento para las futuras generaciones.

Esta afirmación la hace uno de los abuelos quien expresó: "nuestros ancestros no estaban pensando en defenderse de invasores o de colonos, no, ellos más bien estaban regulando y protegiendo el bosque, los ríos, los cerros, porque eran lugares sagrados". Los conversatorios con los abuelos también reafirmaron que los indígenas Mayangnas y Panamahka de Sauni Arungka, siempre respetaron la naturaleza, porque sabían que allí estaba el futuro de sus generaciones. Al respecto una entrevistada señala:

Hoy tengo mucha dificultad para poder explicar cómo vivían nuestros ancestros porque sería estar en contra de mi religión, todo lo que hacían nuestros ancestros era visto como diabólico por mi religión, yo ahora tomo la santa cena en mi iglesia.

Esta afirmación nos indica que el cristianismo llegado con los colonizadores, tanto de España como de Inglaterra, se encargó de borrar muchas tradiciones del pueblo Mayangna. De acuerdo con el conversatorio con los sabios se puede afirmar que la comunicación intercultural desde la cosmovisión indígena Mayangna fue un proceso que permitió una comunicación íntima entre la población ancestral, la Madre Tierra y sus recursos naturales, donde había una armonía entre los seres de la naturaleza y el ser humano, que vivió protegiendo y conservando sus recursos naturales para garantizar su existencia.

Uno de los resultados de este proceso investigativo es que la comunicación que mantuvieron los ancestros con los espíritus de la naturaleza (Espíritu del Bosque, 
Agua, Tierra y Aire) que les dio poderes sobrenaturales, han disminuido de forma drástica debido a que el cristianismo no permite y califica esas prácticas tradicionales de diabólicas. Por ello, el pueblo originario las ha abandonado a cambio de adorar al Dios del Cristianismo.

$\mathrm{Al}$ relacionarlo con lo que plantea Walsh (2005), es evidente que es una práctica de comunicación intercultural que es posible por medio del diálogo entre culturas y que genera una forma de relacionarse ya sea para mantener y conservar prácticas y saberes o para trasnculturalizarlos. De cualquier forma, que se pueda interpretar queda claro para el pueblo originario Mayangna Sauni Arungka, son propios de una comunicación ancestral. Esto es entendido para Ticona (2006), como una forma de cuestionamiento de categorías para poder construir conocimientos interculturales; sin embargo este autor reconoce que el saber indígena y campesino debe ser construido en un pensamiento alternativo integrando el pensamiento propio y crítico, con el propósito de buscar el camino que lleve a la descolonización.

Benedicto Dixon (2015) señala que la comunicación desde sus territorios es vista como un proceso que fortalece el Buen Vivir de los pueblos, los derechos y los deberes, que se sustentan en la práctica del respeto de los individuos. Esta conceptualización es vista desde una perspectiva endógena que tiene como punto de interés el bienestar de los pueblos y su relación con el entorno, lo que favorece relaciones interculturales a través del diálogo e intercambio de saberes. Por ende, y desde la perspectiva de Rodrigo (2011), se entiende como una forma de comunicación para articular y entender las prácticas ancestrales.

Para las autoridades territoriales y población en general el tema de la invasión a sus tierras es el principal problema que tienen en su territorio, porque están destruyendo el bosque y con ello están matando la vida de los pueblos originarios. Implica drásticamente la pérdida de parte de su existencia, pues en ella encuentran sus alimentos, medicinas y también sus áreas de recreación y meditación en los lugares sagrados, como los cerros y ríos. Pese a que la comunicación es un espacio de respeto e intercambio como lo establece Rodrigo (2011), se puede agregar también que es necesario entenderla mostrando reciprocidad y tolerancia.

Martina Juwith Williams (2015) la concibe como aquella comunicación que se da entre sabios, autoridades, niñez y mujeres que forman parte de una cultura en la que se practican costumbres, tradiciones, vivencias, que fortalecen el conocimiento y conservan las culturas ancestrales de los pueblos. Con esta visión, la comunicación intercultural desde la cosmovisión indígena Mayangna Panamahka, está ligada a la búsqueda del consenso, el diálogo, la negociación, el debate, las discusiones, el análisis $y$ el entendimiento. 
Para los indígenas Mayangnas Panamahka, no hay pleito entre ellos, aunque en las discusiones y debates que se llevan a cabo en sus reuniones, se observó una amplia participación de todos los comunitarios que al final llegan a reconocerse como parte intrínseca de la naturaleza y el lenguaje, lo cual orienta hacia una comunicación con identidad desde la espiritualidad y sabiduría de los pueblos.

Las mujeres tienen una forma muy particular de entender el tema de la comunicación ligándola al conocimiento, saberes y aprendizajes que hay en la comunidad sobre la vida, es la forma en que se transfiere el conocimiento a cada generación por medio del ejemplo y enseñar con la práctica. La mujer hace uso de la comunicación intercultural desde el hogar, donde se realizan conversatorios entre la familia, desde los ríos donde conversan los diferentes problemas de la comunidad cuando están lavando la ropa, desde la educación con el ejemplo a los hijos y a las hijas. Esta es la comunicación intercultural que ha venido aportando la mujer desde las comunidades indígenas Mayangna Sauni Arungka.

Después de analizar las entrevistas aplicadas a sabios y sabias se puede inferir que la cosmovisión del pueblo indígena Mayangna Sauni Arungka para la defensa del territorio ha sido una práctica del lenguaje oral. Según Fidencio Davis, la comunicación oral, ha sido la principal forma de comunicación en el pueblo indígena Mayangna, pero que se han auxiliado de algunos instrumentos que existen para comunicarse entre comunidades usando el sonido. Pero también, han usado las asambleas comunitarias que fueron llamadas por sus ancestros como Asang Lawana, hoy ese término está siendo utilizado para la Gran Asamblea de todos los territorios indígenas Mayangna. También lo utiliza la organización que hoy se conoce como la Nación Mayangna, que representa a este grupo indígena de Nicaragua.

Esta afirmación coincide con lo expresado por Eulogio Pedro (2015) quien refiere que la comunicación oral fue fundamental y la palabra era algo que se respetó durante toda la existencia de su pueblo, habían Ditalyan que eran los líderes con poderes sobrenaturales que podían comunicarse con los otros Ditalyan de los demás territorios y esa también fue parte del legado de comunicación que tienen como pueblo. Se utilizaron señales porque sus ancestros han recorrido gran parte del territorio nacional, por ello es que la toponimia de Nicaragua está relacionada con nombre Mayangnas, en ese caminar se fueron dejando señales para que no se perdieran, los cerros los ríos han sido también parte de esa comunicación que les ha acompañado desde siempre al pueblo indígena Mayangna. El uso del Wau una especie de pito que era usado para controlar las intervenciones en las Asang Lawana o asambleas que se realizaban.

Según Armando Edwin Juwith (2015) la comunicación en sí para los pueblos antes era vital, es vista como una herramienta principal de los pueblos, comunicándose en lo que pasa en su entorno, se trata de una expresión oral y se daba entre los Ditalyan o caciques que tenían amplios poderes y podían descubrir cuando uno de sus pueblos 
tenía problemas. La comunicación se daba por los ríos que servían como formas de comunicación, la naturaleza es todo para el pueblo indígena. También por lo espiritual, porque se comunicaban con los espíritus de Dikutna, del duende, ellos tenían el poder de avisar al otro Ditalyang. El Wau wau era un instrumento que se daba y se usaba para emitir un sonido que tenía un mensaje, la señal en este caso como algo biológico o abiótico, el viento si venía en una dirección el pueblo manejaba eso, y se entendía la naturaleza que les avisaba de lo que iba a pasar, pero el cambio climático ha venido afectando esa comunicación con la naturaleza, debido al daño que se ha hecho a la madre tierra.

Mario Peralta Bans (2015) afirma que se ha usado el fuego y el humo, que era algo que se comunicaban de esa forma, el humo era una señal de que alguien andaba perdido y que se debía ir a buscar. Cuando se caminaba se dejaban señas en forma de cruz en el camino y eso significaba que allí hubo un robo en una milpa. Un elemento simbólico de comunicación estaba expresado en el uso de un material natural que emitía sonido que era usado para convocar a las reuniones de los comunitarios. Actualmente se utiliza la campana de las iglesias para hacer la misma convocatoria. La comunicación era oral, pero también se usó las señas, pero si alguien de la familia sabía escribir se usaba por medio de carta. Esto evidencia que existe un acercamiento entre naturaleza y espiritualidad humana propia de los pueblos.

Otra forma de percibir y entender la comunicación intercultural desde la cosmovisión indígenas Mayangna es la Asang Lawana, allí se reunían los Ditalyan que eran personas superdotadas con poderes especiales y realizaban sus rituales a la orilla de los cerros, no podían llegar a esa reunión los comunitarios común y corriente. Esas reuniones se hacían a la orilla de los cerros, no llegaba cualquier persona, se tomaban decisiones que se expresaban en una comunicación de forma oral y la palabra era de gran valor, allí se reunían los caciques y otras personas muy importantes.

Venían y después esas decisiones eran comunicadas oralmente, no había estatutos, ni nada. Pero se abordaban temas relacionados a la tierra y al territorio que eran considerado como el supermercado, la farmacia porque se sacaban medicamentos, era un lugar de teatro porque se hacían ejercicios en los ríos, o cuando se reunían en las faldas de los ríos se hacían rituales, era un lugar de recreación y también se usaba como iglesias, habían casamientos entre el hombre y la mujer, en esa Asang Lawana también se hacían matrimonios. Para los abuelos y abuelas con los que se conversó la defensa del territorio ha estado de forma permanente en el pueblo indígena Mayangna, con la diferencia que antes no había muchas invasiones como desde 1990 en que se viene presentando este fenómeno. 
Se puede destacar que para desarrollar una comunicación intercultural desde los pueblos Panamahka es necesario que se practique una lengua común, un reconocimiento de la cultura ajena, el reconocimiento de la cultura propia, tener una relación equilibrada y que ésta se vuelve recíproca cuando la práctica es equilibrada desde las culturas de los pueblos.

\section{Conclusiones}

A partir de la investigación realizada por medio de los instrumentos aplicados en el territorio, se puede afirmar las conclusiones siguientes:

La comunicación intercultural de los ancestros del Pueblo Indígena Mayangna Sauni Arungka está ligada a la comunicación íntima con los espíritus del agua, aire, tierra y bosque, eso les daba poderes sobre naturales principalmente a los Ditalyang.

La comunicación intercultural del pueblo indígena Mayangna Sauni Arungka, estaba también muy ligada a la conservación de todo lo que había en el territorio y por ello establecían normas para la conservación y protección de la flora y fauna. Los Ditalyang fueron obligados a abandonar sus prácticas tradicionales por el cristianismo.

Hoy la preocupación de los líderes del gobierno territorial indígena y la población en general está centrada en la incursión de personas ajenas a su cultura que han invadido sus tierras, por lo que apuestan con el diálogo a buscar una salida al conflicto. De allí, la importancia en enfatizar que la comunicación intercultural de las nuevas generaciones apuesta a ese diálogo de saberes, entre los invasores y los pueblos originarios, al entendimiento y al consenso.

Concluimos diciendo, que se hace necesario diseñar un proyecto de formación orientado a impartir un diplomado en comunicación intercultural comunitaria para facilitar herramientas que les permita a la comunidad poder incidir desde las herramientas tecnológicas a nivel nacional e internacional. Asimismo, gestionar los recursos económicos necesarios para que desde la Universidad de las Regiones Autónomas de la Costa Caribe Nicaragüense, se pueda ejecutar acciones de formación para jóvenes comunicadores del territorio indígena.

\section{Lista de referencias}

Boas, F. (2008). Antropología, Editorial Centro de Estudios Ramón Areces, Madrid, 2008.

Cuningham, M. (2008). Espiritualidad, Conocimiento e Historia de Pueblos Indígenas de Abya Yala. Nicaragua. 
Calero \& Larios (2014). Cosmovisión Amazónica. Recuperado en: http://es.slideshare. net/asrose/cosmovisión-amazónica.

Cox, A. (2011). El vuelo del alma. Managua.

Durkheim (1997). Las reglas del método sociológico. México.

Fedrick, J. (2003). Información sobre los Pueblos Indígenas de Nicaragua para el proyecto regional manejo integrado de ecosistemas por pueblos indígenas y comunidades de Centroamérica. Managua.

Geertz, C. (1994). Conocimiento local: ensayos sobre la interpretación de las culturas.

Marín, G. (2014). Espiritualidad y cosmovisión indígena. Recuperado en: http://www.toltecayotl.org/tolteca/index.php/2014-03-30-23-46-16/biblioteca-tolteca/10689iii-espiritualidad-y-cosmovisión-indigena-griselda-medina-villca

Prieto, D. \& Vásquez, A. (20014). Cosmovisión amazónica. México.

Rodrigo, M. (2011). La Comunicación Intercultural. Recuperado en: www.portalcomunicacion.com/download/1.pdf

Ruíz L. (2010). Economía y relaciones interétnicas Mayagna Sikilta, Siuna RAAN. Tesis Doctoral.

Silva, R. (2015). Bolivia, cosmovisión indígena y revolución. Recuperado en: http://caio. uy.over-blog.com/article-bolivia-cosmovisión-indigena-y-revolucion-55122109. html

Ticona , E. (2006). Conferencia sobre cátedra indígena. Cochabamba, Bolivia.

URACCAN (2009). Política de Comunicación Intercultural de la Universidad de las Regiones Autónomas de la Costa Caribe Nicaragüense. Managua, Nicaragua.

Walsh, C. (2005). Interculturalidad, poder, conocimiento, colonialismo, capitalismo global. Revista Javeriana, 50. 